\title{
ERRATUM
}

\author{
Ayato Kohzu • Ichiro Tayasu $\cdot$ Chikage Yoshimizu \\ Atsushi Maruyama $\cdot$ Yukihiro Kohmatsu \\ Fujio Hyodo · Yukio Onoda • Akitake Igeta \\ Kiyoshi Matsui · Takanori Nakano • Eitaro Wada \\ Toshi Nagata $\cdot$ Yasuhiro Takemon

\section{Nitrogen-stable isotopic signatures of basal food items, primary consumers and omnivores in rivers with different levels of human impact}

Published online: 21 June 2008

(C) The Ecological Society of Japan 2008

\section{Erratum to: Ecol Res \\ DOI 10.1007/s11284-008-0489-x}

In this article the \% symbol should have been used instead of the \% symbol except in the first paragraph of the "Results" section and in all occurrences of terms such as "\%paddy", "\% residential" and "\% orest", where it was used correctly.

The online version of the original article can be found under doi:10.1007/s11284-008-0489-x.

A. Kohzu · C. Yoshimizu

Japan Science and Technology Agency,

509-3 Hirano-Nichome, Otsu, Shiga 520-2113, Japan

A. Kohzu · I. Tayasu $\cdot$ C. Yoshimizu $\cdot$ Y. Onoda · T. Nagata

Center for Ecological Research, Kyoto University,

509-3 Hirano-Nichome, Otsu, Shiga 520-2113, Japan

\section{A. Maruyama}

Department of Environmental Solution Technology, Ryukoku University, 1-5 Yokotani, Seta Oe-cho,

Otsu 520-2194, Japan

Y. Kohmatsu $\cdot$ F. Hyodo $\cdot$ A. Igeta $\cdot$ T. Nakano $\cdot$ E. Wada Research Institute for Humanity and Nature,

457-4 Motoyama, Kamigamo, Kita-ku,

Kyoto 603-8047, Japan

K. Matsui

School of Science Education,

Nara University of Education, Takabatake-cho,

Nara 630-8528, Japan

\section{Y. Takemon}

Disaster Prevention Research Institute,

Kyoto University,

Gokasho, Uji 610-0011, Japan
Present address: A. Kohzu ( $ه$ )

Lake Environment Research Section,

National Institute for Environmental Studies,

16-2 Onogawa, Tsukuba, Ibaraki 305-8506, Japan

E-mail:kohzu@nies.go.jp

Tel.: + 81-29-8502187

Fax: + 81-29-8502576

Present address: E. Wada

Frontier Research Center for Global Change,

Japan Agency for Marine-Earth Science and Technology,

3173-25 Showamachi, Kanazawa-ku,

Yokohama 236-0001, Japan

Present address: T. Nagata

Ocean Research Institute, University of Tokyo,

1-15-1 minamidai, Nakano-ku,

Tokyo 164-8639, Japan 